\title{
The Treatment of Non-Melanoma Skin Cancer with Image-Guided Superficial Radiation Therapy: An Analysis of 2917 Invasive and In Situ Keratinocytic Carcinoma Lesions
}

Lio Yu $\cdot$ Chad Oh $\cdot$ Christopher R. Shea

Received: September 19, 2020 / Accepted: January 5, 2021 / Published online: February 5, 2021

(C) The Author(s) 2021

\section{ABSTRACT}

Introduction: An image-guided form of superficial ionizing radiation therapy (IGSRT) is becoming a commonly used alternative to surgery for non-melanoma skin cancer (NMSC). However, there is little literature evidence evaluating the efficacy and safety of this approach. This study evaluates the efficacy and safety of IGSRT in treating a large number of patients with NMSC.

Supplementary Information The online version contains supplementary material available at https:// doi.org/10.1007/s40487-021-00138-4.

L. Yu (凶)

Radiation Oncology, Laserderm Dermatology, Smithtown, NY, USA

e-mail: lio.yu@protonmail.com

L. Yu

SkinCure Oncology, Burr Ridge, Illinois, USA

C. Oh

The Weinberg Group, Washington, D.C., USA

C. R. Shea

Dermatology, University of Chicago Medicine, Chicago, IL, USA
Methods: The medical records of 1632 stage 0-II patients with 2917 invasive and in situ NMSC lesions treated from years 2017 to 2020 were reviewed. No patients had clinical evidence of regional lymph node or distant disease at presentation.

Results: Treatment, guided by pre-treatment ultrasound imaging to adjust radiation energy and dose, combined with a fractionation treatment schedule of 20 or more treatment fractions, was safe and well tolerated. Of 2917 NMSC lesions treated, local tumor control was achieved in 2897 lesions, representing a $99.3 \%$ rate of control.

Conclusion: IGSRT should be considered as a first-line option for treating NMSC tumors in suitable early stage patients. Cure rates observed in this initial period of follow-up are similar, and potentially superior with further follow-up, to traditional superficial radiation therapy (SRT) and surgical options.

Keywords: Basal cell carcinoma; Image-guided superficial radiation therapy; Non-melanoma skin cancer; Squamous cell carcinoma; Squamous cell carcinoma in situ 


\section{Key Summary Points}

Image Guided Superficial Radiation Therapy (IGSRT) was safe and well tolerated in this study, involving 1632 patients with non-melanoma skin cancer (NMSC). Of 2917 NMSC lesions treated, local tumor control was achieved in 2897 lesions, representing a $99.3 \%$ rate of control.

IGSRT should be considered as a first-line option for treating stage 0-II NMSC lesions in suitable patients, especially those who are not candidates for surgery or who decline surgery.

NMSC is a highly prevalent condition, with an estimated annual incidence in the U.S. of 5.5 million tumors in 2012.

This study presents a retrospective evaluation of efficacy and safety of imageguided superficial radiotherapy, a nonsurgical treatment option, in 2917 NMSC lesions (1632 patients).

Treatment with IGSRT resulted in local tumor control in 2897 lesions, representing a $99.3 \%$ rate of control.

IGSRT was safe and well-tolerated in this study.

These results suggest IGSRT should be considered as a first-line option for treating NMSC in suitable patients.

\section{DIGITAL FEATURES}

This article is published with digital features, including a summary slide, to facilitate understanding of the article. To view digital features for this article go to https://doi.org/10.6084/ m9.figshare.13521686. 
patients with poor general health, bleeding tendency, etc.) [11]. However, more recent studies of patients treated in the outpatient setting have shown a higher rate of control of NMSC [12, 13]. The 2020 National Comprehensive Cancer Network (NCCN) Guideline states that for basal cell and squamous cell skin cancers, "[s]urgical approaches often offer the most effective and efficient means for accomplishing cure, but considerations of function, cosmesis, patient preference, and performance status may lead to choosing RT [radiation therapy] as primary treatment in order to achieve optimal overall results" [14, 15].

There are several potential explanations for the discrepancies in the published literature regarding the efficacy of SRT to treat NMSC. First, the setting in which SRT is administered to treat NMSC has changed over the years. Many decades ago, dermatologists often provided SRT in their offices, but this practice pattern changed to the point where SRT came to be rarely offered in the outpatient dermatology setting. While there has subsequently been a substantial resurgence in the use of SRT in dermatologist's offices in the USA, it remains less common today than in past times [16]. Thus, much of the SRT literature concerns the particular patient population referred out to radiation oncologists; that is, when the patient presents with NMSC considered not amenable to treatment by dermatologists [10]. This circumstance introduces potential selection bias, in that NMSC cases referred for SRT may have an increased proportion of larger or more aggressive tumors with a higher risk of recurrence [16-18].

Change in the technique of SRT may lead to further developments in its demonstrated efficacy to treat NMSC. Recent years have seen technical advances in the delivery of SRT, including image-guided approaches made more readily available through inclusion of high-frequency ultrasound capabilities with SRT delivery technology [19]. An ultrasound unit specifically designed to detect dermatologic structures using frequencies of $22 \mathrm{MHz}$ (which is ideal for visualizing superficial skin depths of 0-6 $\mathrm{mm}$ ) has been integrated with the commercial IGSRT unit (Sensus Healthcare). This allowed generally easy determination and verification of the tumor depth as well as lateral extent of the lesion. Knowing the depth of the tumor and correlating with the percentage depth dose (PDD) tables of the IGSRT machine from the manufacturer allow the optimal choice of energy $(50,70$, or $100 \mathrm{kV})$ to be selected before and during the course of treatment, allowing adjustments to be performed accordingly. This paper reports experience with an image-guided form of SRT (IGSRT) in a large group of patients with NMSC for whom IGSRT was used. The study cohort represents a subset of data (Texas location only) presented by $\mathrm{Yu}$ et al. at the May 2020 Annual Meeting of the Society for Investigative Dermatology (SID) [20]. This data was combined with data collected and analyzed from similarly treated NMSC lesions in South Carolina and two sites in Indiana ( $\mathrm{Yu}$ et al. manuscripts in preparation). Our findings indicate that application of IGSRT results in an excellent rate of cure, and that IGSRT is a highly effective first-line treatment method for NMSC.

\section{METHODS}

\section{Patients}

A direct chart analysis of 1632 patients with a histopathologic diagnosis of NMSC treated with IGSRT was performed. Diagnosis and staging of lesions had been completed prior to treatment on the basis of a tissue biopsy performed by a dermatologist at each site. The dermatologist referred patients for IGSRT on the basis of patient and lesion characteristics, which included pathologic confirmation of SCC, BCC, or SCC in situ (SCCIS), the latter most often with suspicion of invasion or full-thickness atypia. The ultimate decision of referral for IGSRT was determined by individual providers. Treatment data and lesion characteristics at the time of treatment were collected retrospectively. The medical records of 702 female and 930 male patients (mean age $73.2[\mathrm{SD} \pm 10.93]$ years) with 2917 lesions treated from years 2017 to 2020 were retrospectively reviewed. Every lesion was considered as an independent cancer 
lesion. All patients included in this analysis were at stage grouping 0 , I, or II (i.e., Tis, T1, or $\mathrm{T} 2$ without clinical evidence of regional lymph node or distant disease (NO and M0)) at presentation, based on the American Joint Committee on Cancer (AJCC) 8th Ed. Cancer Staging Manual [21]. While AJCC 8th Ed. staging pertained to cutaneous SCC of the head and neck, these same criteria for staging were applied in this study to all BCC, SCC, and SCCIS lesions throughout the entire body to be consistent. Follow-up interval and data were initially gathered manually from written and electronic medical records. Subsequent updates to patient follow-up intervals were accessed electronically with the assistance of algorithmic analysis provided by a healthcare data company (Sympto Health, Inc.).

The authors adhered to the principles established in the Federal Policy for the Protection of Human Subjects, referred to as the "Common Rule," as well as the pertinent sections of the Helsinki Declaration and its amendments. The data have been de-identified for use in this study. All patients gave informed consent before treatment.

\section{Treatment Guidelines}

All lesions received IGSRT, consisting of energies of 50,70 , or $100 \mathrm{kV}$ based on a standardized, proprietary protocol developed by a boardcertified radiation oncologist in conjunction with a board-certified dermatological surgeon. There are a wide range of dose regimens to choose from that have been established over decades of radiation therapy treatment depending on situations, ranging from total fractions of less than 5 to more than 35 and daily doses ranging from 120 cGy (twice a day) to 2250 cGy $[22,23]$. For curative treatments of all tumors (not just skin) the most common present-day regimen employed by radiation oncologists is $180-200$ cGy given daily five times per week for 30-36 treatments to a dose of 5400-7200 cGy.

Skin treatments involving small fields are amenable to a hypofractionated regimen of 2-4 times per week at higher "daily" fractions of (generally) 220-400 cGy. A common regimen amongst radiation oncologists experienced with head, neck, and skin cancer treatments uses 20 fractions of $250-260 \mathrm{cGy} .{ }^{1}$ This "middle of the road" regimen was felt by some radiation oncologists/dermatologists to deliver the optimum in cure, cosmesis, and convenience while minimizing complications (Yu et al. Annual SID Meeting, May 2020). The specific treatment prescription for each patient was determined by the treating clinician not involved with this study, which is a retrospective, observational review of this "real-world" evidence. A mean total treatment dose of 5219.9 $(\mathrm{SD} \pm 224.47) \mathrm{cGy}$ was given in the entire group. The energy administered to each tumor was selected on the basis of that particular tumor's characteristics, including anatomic location, histogenetic type, lesion depth, and skin curvature. In 2017 and 2018, the treatment guideline in use recommended their "most common protocol" of 255 cGy $\times 20$ total fractions with either 50 or $70 \mathrm{kV}$ (based on lesion depth less than or greater than $1.5 \mathrm{~mm}$, respectively) given three times a week. Tumors less than $2 \mathrm{~cm}$ or greater than $4 \mathrm{~cm}$ were treated four times per week at $245 \mathrm{cGy}$ or $240 \mathrm{cGy}$ for 20 or 25 fractions, respectively. Lesions below the knee were optionally treated five times a week at $180 \mathrm{cGy}$ for 30 fractions.

Starting in 2019, a more detailed proprietary protocol with a new guidance table developed by Dermatology and Radiation Oncology (Ladd$\mathrm{Yu}$ table) was employed incorporating specific $\mathrm{TDF}^{2} /$ dose/fractionation/energy recommendations based on ultrasound depth and tumor type. This modified the "most common

\footnotetext{
1 The IGSRT machine is not designed to deliver doses based on centiGray but instead based on time (minutes) the beam is turned on to 2 decimal points. For example, a field of $2 \mathrm{~cm}$ diameter giving $70 \mathrm{kV}$ for $0.42 \mathrm{~min}$ will deliver a dose of $271.74 \mathrm{cGy}$, whereas a time of $0.43 \mathrm{~min}$ would deliver a dose of $278.21 \mathrm{cGy}$. Therefore, it is nearly impossible for a guideline protocol to mandate an exact dose per fraction of, say, $255.0 \mathrm{cGy}$ but rather, depending on the "beam on time," the treatment dose that comes closest to that dose is generally selected.

2 Time dose fractionation (TDF) is a useful system of tables representing biologic equivalent doses often used by radiation oncologists/therapeutic radiologists specifically for treatment of skin cancers with different fractionation schedules.
} 
protocol" to a fractionation dose range of 245-279 cGy for 20 fractions three or four times per week to achieve a therapeutic biological dose range of 90-99 or greater TDF using 50, 70, or $100 \mathrm{kV}$ energy (see Table S1 in the electronic supplementary material). Larger, deeper, and high-risk legions were recommended higher doses per fraction and/or more fractions (greater than or equal to 22) to achieve higher biologically effective doses. Providers/therapists were encouraged to consult with the national radiation oncologist and/or chief medical officer during weekly grand rounds or through email discussions in these situations. These treatment protocols were initially developed by a boardcertified radiation oncologist and further refined by a dermatologic surgeon (Dr. Ladd) in collaboration with another board certified radiation oncologist (Dr. $\mathrm{Yu}$ ) on the basis of sound radiation and dermatologic treatment principles and generally conform to the biological dose ranges suggested by the NCCN for cutaneous BCC and SCC [14, 15]. In retrospect, the independently derived hypofractionated regimens are consistent with current ASTRO Clinical Practice Guideline for cutaneous SCC and BCC [24]. Pre-treatment ultrasound imaging was performed prior to delivery of each treatment fraction to evaluate lesion depth and configuration. Adjustments to prescribed energy and daily dose were made on the basis of ultrasound findings to minimize radiation exposure, optimize clinical outcomes, and reduce potential toxicity. The percentage changes in energy (mixed energies) during the course of treatment were collected to reflect the impact of image guidance on the treatment.

The treatment fractions were administered by a radiation therapist at a frequency of 3,4 , or 5 times per week, based on the discretion of the treating practitioner and in accordance with standardized treatment protocols as discussed above which are consistent with typical radiation oncology recommendations for treatment of small early stage neoplasms in curative, nonpalliative cases where cosmesis is of importance. Patients treated with a range of 20-30 fractions were included. Patients falling out of this guideline range were excluded from this analysis. Medical physics consultations were also provided during the course of treatment. A median of 20 fractions were delivered, and the mean follow-up was 69.8 weeks (range 0-220.9 weeks).

\section{Study Endpoints}

The endpoints include the absence of local evidence of disease at follow-up visits and Radiation Treatment Oncology Group (RTOG) toxicity, including the most common toxicities and their severity [25]. RTOG toxicity was prospectively documented in the charts routinely after every 5-fraction increment and these findings were extracted and documented as the highest RTOG grade throughout the entire course of treatment.

\section{Statistical Analysis}

Demographic and baseline characteristics, original lesion depth, tumor staging, treatment information, lesion response, survival status, and RTOG toxicity levels were summarized descriptively. All lesions meeting inclusion criteria were included and summaries provided by patient and lesion. The duration of follow-up was summarized and defined as the date of last follow-up minus date of completion plus 1 day and then converted to both weeks and months.

Summary statistics for continuous outcomes included the number of observations $(n)$, mean, standard deviation, minimum, median, and maximum while categorical outcomes were summarized with frequency and percentages. Missing data were not imputed and Fisher's exact tests were performed to evaluate significant differences between histologies. SAS v9.4 was used for all analyses.

\section{RESULTS}

A total of 2917 NMSC lesions treated with IGSRT from 2017 to 2020, consisting of 1406 BCC, 920 SCC, and 603 SCCIS lesions (certain lesions had combinations of two or more of these histologies), were retrospectively reviewed. Clinical efficacy was evaluated in a 
Table 1 Anatomic distribution of NMSC lesions

\begin{tabular}{ll}
\hline Head and neck (H\&N) & $1692 / 2917(58.0 \%)$ \\
H\&N sublocation ${ }^{a}$ & \\
Ear & $270 / 2917(9.3 \%)$ \\
Cheek & $403 / 2917(13.8 \%)$ \\
Nose & $479 / 2917(16.4 \%)$ \\
Cutaneous lip & $71 / 2917(2.4 \%)$ \\
Mucosal lip & $10 / 2917(0.3 \%)$ \\
Forehead & $265 / 2917(9.1 \%)$ \\
Temple & $69 / 2917(2.4 \%)$ \\
$\quad$ Neck & $110 / 2917(3.8 \%)$ \\
Extremities & $615 / 2917(21.1 \%)$ \\
Trunk & $182 / 2917(6.2 \%)$ \\
Hand & $140 / 2917(4.8 \%)$ \\
Back & $128 / 2917(4.4 \%)$ \\
Shoulder & $86 / 2917(2.9 \%)$ \\
Chest & $81 / 2917(2.8 \%)$ \\
\hline
\end{tabular}

Lesion locations not exclusive

${ }^{\text {a }}$ Other $\mathrm{H} \& \mathrm{~N}$ sublocations $=15$

total of 1632 patients. Patients were predominantly men $(57 \%)$. The mean age of patients was $73.2(\mathrm{SD} \pm 10.93)$ with the range from 31.8 to 101.4 when they received the first IGSRT.

Table 1 demonstrates the anatomic distribution of NMSC lesions. The most common sites of the lesions were head and neck as a group $(58.0 \%)$, extremities $(21.1 \%)$, followed by the head and neck subgroups nose (16.4\%) and cheek (13.8\%).

Table 2 demonstrates cancer cell types and initial lesion size at initiation of therapy. BCC (48.2\%) and SCC (31.5\%) were more common than SCCIS $(20.7 \%)$. The mean diameter of measured lesions was $1.2 \mathrm{~cm} \quad(\mathrm{SD} \pm 0.65)$, ranging from 0.0 to $5.5 \mathrm{~cm}$. The mean depth was $1.2 \mathrm{~mm}(\mathrm{SD} \pm 0.52)$ for $\mathrm{BCC}, 1.2 \mathrm{~mm}$ $(\mathrm{SD} \pm 0.60)$ for $\mathrm{SCC}$, and $1.0 \mathrm{~mm}(\mathrm{SD} \pm 0.41)$ for SCCIS.

Table 3 shows lesions by diameter and histology. Of note, eight lesions greater than or equal to $4 \mathrm{~cm}$ were all SCCIS.
Table 2 Cancer types and initial lesion size

\begin{tabular}{|c|c|}
\hline Cancer types ${ }^{\mathrm{a}}$ & $n=2917$ \\
\hline $\mathrm{BCC}$ & $1406 / 2917(48.2 \%)$ \\
\hline SCC & $920 / 2917(31.5 \%)$ \\
\hline SCCIS & $603 / 2917(20.7 \%)$ \\
\hline Lesion diameter $(\mathrm{cm})$ at start & $n=2892$ \\
\hline Mean $\pm S D$ & $1.2 \pm 0.65$ \\
\hline Range & $0.0-5.5$ \\
\hline Median & 1.0 \\
\hline BCC lesion depth $(\mathrm{mm})$ at start & $n=1303$ \\
\hline Mean $\pm S D$ & $1.2 \pm 0.52$ \\
\hline Range & $0.0-4.8$ \\
\hline Median & 1.2 \\
\hline SCC lesion depth $(\mathrm{mm})$ at start & $n=855$ \\
\hline Mean $\pm S D$ & $1.2 \pm 0.60$ \\
\hline Range & $0.0-4.4$ \\
\hline Median & 1.1 \\
\hline SCCIS lesion depth $(\mathrm{mm})$ at start & $n=530$ \\
\hline Mean $\pm S D$ & $1.0 \pm 0.41$ \\
\hline Range & $0.0-3.4$ \\
\hline Median & 0.9 \\
\hline
\end{tabular}

${ }^{a}$ Certain lesions had combinations of multiple histologies

Table 4 summarizes the total number of treatments, total treatment dose, duration of treatment, and follow-up interval. The mean total number of fractions was $20.1(\mathrm{SD} \pm 0.71)$ with the range from 20 to 30 . The mean total treatment dose was 5219.9 cGy ( $\mathrm{SD} \pm 224.47)$, with the range from 3716.0 to $7363.7 \mathrm{cGy}$. All lesions were treated with energies ranging from 50 to $100 \mathrm{kV}$. The majority of these lesions were treated for 6-7 weeks and followed for a mean of approximately 69.8 weeks.

Among 1632 patients, 1612 patients (98.8\%) did not have evidence of disease at their last follow-up visit, and 20 patients (1.2\%) had evidence of disease at their last follow-up visit. Specific to individual lesions, of the 2917 lesions that underwent IGSRT treatment, 2897 lesions $(99.3 \%)$ did not have evidence of disease 
Table 3 Diameter distribution of NMSC lesions

\begin{tabular}{llllll}
\hline Diameter $^{\mathbf{a}}$ & BCC and SCC & BCC only & SCC only & SCCIS only & Total \\
\hline 0 to $<2 \mathrm{~cm}$ & 8 & 1167 & 752 & 516 & 2427 \\
2 to $<4 \mathrm{~cm}$ & 3 & 236 & 164 & 60 & 457 \\
$\geq 4 \mathrm{~cm}$ & 0 & 0 & 0 & 8 & 8 \\
\hline
\end{tabular}

${ }^{a}$ Missing values $=25$

Table 4 Treatment fractions, dose, duration, and follow-up interval

\begin{tabular}{lll}
\hline Characteristic & Statistic & $(\boldsymbol{n}=\mathbf{2 9 1 7})$ \\
\hline Total number of treatments & $N$ & 2917 \\
& Mean \pm SD & $20.1 \pm 0.71$ \\
Total treatment dose (cGy) & Range & 20.0 to 30.0 \\
& Median & 20.0 \\
& $N$ & 2917 \\
Duration of treatment (weeks) & Mean \pm SD & $5219.9 \pm 224.47$ \\
& Range & 3716.0 to 7363.7 \\
Duration of follow-up (weeks) & Median & 5188.4 \\
& $N$ & 2917 \\
& Mean \pm SD & $7.1 \pm 1.55$ \\
& Range & $4.0-22.6$ \\
& Median & 6.7 \\
& $N$ & 2917 \\
& Mean \pm SD & $69.8 \pm 54.62$
\end{tabular}

at their last follow-up visit. There were 20 lesions $(0.7 \%)$ that did not respond completely to IGSRT or recurred.

Among 1632 patients, 1591 patients (97.5\%) were alive as of October 2020 and 41 patients (2.5\%) had died. All deaths were deemed unrelated to the treatment of NMSC by IGSRT. This death rate is not substantially different from the general population in the same age group [26].

Table 5 demonstrates the treatment by energy. Of note, 847 lesions were treated with a mix of two or more energies, representing $29 \%$ of the lesions.
Table 6 summarizes the safety results by lesion. Among 2177 lesions with RTOG toxicity grade data available, only 20 lesions $(0.9 \%)$ were RTOG grade 3 or 4 in severity.

\section{Subgroup Analysis}

\section{All Lesions with More Than 12 Months of Follow-up}

The cohort with greater than 12 months of follow-up (mean $107.7 \pm 41.31$ weekly) was analyzed (1639 lesions). There were no substantial 
Table 5 NMSC lesion distribution by energy

\begin{tabular}{llllll}
\hline Energy $(\mathbf{k V})$ & BCC only & SCC only & SCCIS only & Combined BCC and SCC & Number of lesions $^{\mathbf{a}}$ \\
\hline 50 & 818 & 683 & 554 & 10 & 2165 \\
70 & 828 & 524 & 124 & 4 & 1480 \\
100 & 50 & 64 & 0 & 0 & 114 \\
Mixed & 400 & 364 & 80 & 3 & 847 \\
\hline
\end{tabular}

a Energy classification not exclusive

Table 6 Safety-by lesion

\begin{tabular}{lllc}
\hline Characteristic & Grade & Description & $(\boldsymbol{n}=\mathbf{2 1 7 7})$ \\
\hline $\begin{array}{l}\text { Highest RTOG toxicity } \\
\text { grade }\end{array}$ & 1 & Follicular, faint, or dull erythema; epilation; dry desquamation; & $1717 / 2177$ \\
& & decreased sweating & $(78.9 \%)$ \\
& 2 & Tender or bright erythema, patchy moist desquamation; moderate & $440 / 2177$ \\
& & edema & $(20.2 \%)$ \\
& 3 & Confluent, moist desquamation other than skin folds; pitting edema & $16 / 2177(0.7 \%)$ \\
& 4 & Ulceration, hemorrhage, necrosis & $4 / 2177(0.2 \%)$ \\
\hline
\end{tabular}

RTOG Radiation Treatment Oncology Group

differences (greater than 5\%) in this cohort compared to the entire group in terms of age, gender, age at first treatment, tumor location, size of lesion, and tumor stage. This subgroup had $5.8 \%$ fewer BCCs $(42.4 \%)$ than the entire group, where BCC comprised $48.2 \%$ of cases. In this cohort, the median of treatments remained at 20 with a mean treatment dose of 5175 cGy $(\mathrm{SD} \pm 215.80)$ with a range of 3716.0$6797.7 \mathrm{cGy}$. While the percentage of mixed energy use remained the same at $29 \%$, this subgroup cohort had approximately $10 \%$ higher utilization at $50 \mathrm{kV}$ energy (84.1\%) compared to the entire group (74.2\%). Use of $70 \mathrm{kV}$ and $100 \mathrm{kV}$ in this subgroup was $43.2 \%$ and $1.3 \%$ compared to the entire group at $50.7 \%$ and $3.9 \%$, respectively. The percentage of disease control by lesion was essentially identical in this subgroup compared to the entire group (1647 of 1659 (99.3\%) vs 2897 of 2917 (99.3\%) lesion control). Survival percentages (again unrelated to tumor control) were similar at $97.6 \%$ (872 of 893 ) vs $97.5 \%$ (1591 of 1632 ). RTOG lesion toxicity was similar with less than $2 \%$ difference at every grade level.

\section{Subgroup of All BCC and SCC Lesions Excluding SCCIS with More Than 12 Months Follow-up}

We ascertained the results where SCCIS (stage 0) lesions were eliminated and found that the entire (invasive cancer only) group had essentially the same tumor response by lesions (99.2\%). In this invasive BCC/SCC-only subgroup with follow-up greater than 12 months, the results were virtually unchanged with 751 of 761 (98.7\%) patients and 1232 of 1242 (99.2\%) lesions controlled. Lesion size as well as treatment doses and number of treatments were chiefly unchanged across the board for all subgroups (Table 7).

\section{DISCUSSION}

\section{Efficacy}

The present study documents the results of one of the largest multi-institutional studies of SRT for the treatment with curative intent of primary NMSC of the skin. Of 1632 patients with 


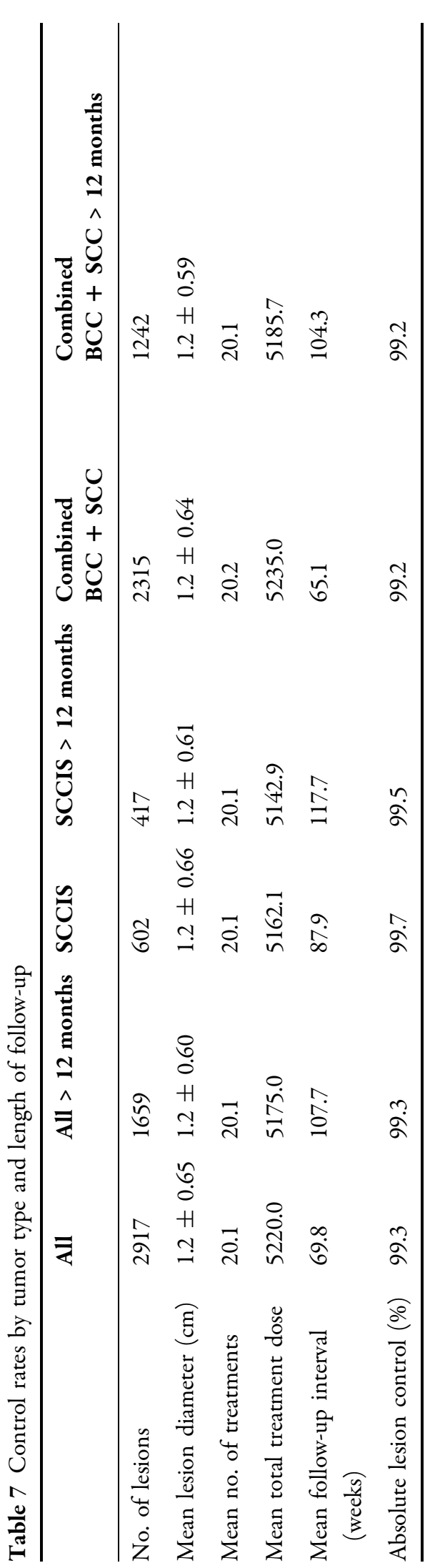

NMSC treated, complete eradication of the tumor was achieved in all but 20 patients, representing a $98.8 \%$ rate of control of the malignancy at the patient level. It should be noted that the control rate by patient indeed underestimates the actual control rate as many patients have more than one lesion that was treated, making the denominator lower in calculations. In fact, the 20 patients with failures had a total of 66 lesions that were treated synchronously or metachronously. A more accurate measure of efficacy of this modality is control rate by lesion. Of 2917 NMSC lesions treated, local tumor control was achieved in 2897 lesions, representing a $99.3 \%$ rate of control. Because $58.0 \%$ of the NMSC lesions treated with IGSRT were located on the anatomically and cosmetically sensitive region of the head and neck, the tissue-sparing property of IGSRT is notable.

These results, while observed with approximately $55 \%$ of patients having follow-up for 12 months or more, appear at this time to be at least consistent with the results of standard surgical and nonsurgical modalities used to treat NMSC. Specifically, the finding of a complete lesion response rate of $99.3 \%$ on early follow-up for IGSRT compares favorably with that of a study of SRT by Hernández-Machin et al., reporting that SRT treatment of 710 patients led to 5-year cure rates of $94.4 \%$ for BCC and $92.7 \%$ for SCC, respectively. The authors concluded that SRT was effective for BCC and SCC and could be a suitable first option in many cases [12]. The response rate observed in our study cohort also seems consistent with and, with further follow-up, has the potential to exceed that reported in a study of 1149 patients by Cognetta et al., who found that SRT-treated patients had mean cumulative recurrence rates for BCC of $2.0 \%$ and $4.2 \%$, and for SCC of $1.8 \%$ and $5.8 \%$, at 2 and 5 years, respectively [13]. The findings of this analysis are in concordance with the previous conclusions presented by $\mathrm{Yu}$ et al., with this analysis of the IGSRT data subset using separate statistical software (SAS) by an outside third-party data analysis company (Summit Analytical). A limitation of the results presented here is the shorter period of follow-up (mean follow-up of 
$69.8 \pm 54.62$ weeks) compared to these earlier evaluations of SRT. Continued follow-up with these patients to 2 and 5 years is expected to yield additional observations relevant to the use of IGSRT.

In total, $1.2 \%(n=20)$ of our patients, representing $0.7 \%(n=20)$ of NMSC lesions, failed to achieve local tumor control with IGSRT. An evaluation of demographic and lesion characteristics for these patients was performed to examine any potential commonalities, including histopathology, lesion size and location, and patient demographics. This evaluation did not identify any significant predictor for patient non-responsiveness to IGSRT at this time. We observed that the non-response rate for SCCIS was $0.3 \%(n=2)$ versus that for BCC $(n=13$, $0.9 \%)$ versus SCC $(n=5,0.6 \%)$. There was no significant difference in control rates between histologies $(p>0.05)$ by Fisher's exact tests. We feel that an explanation of this non-significant difference between differing histologies may, in part, be due to the uniformly excellent control rate achieved in all subgroups with this IGSRT treatment. This suggests that IGSRT is effective in all subsets of NMSC on early follow-up. If this trend continues, a potential advantage of IGSRT in treatment of SCC over other older SRT techniques and MMS may become apparent with longer follow-up. In addition, 41 patients died during the course of the study. These deaths were determined not to be related to their NMSC treatment and had no impact on local control in this study. Indeed, NMSC are generally considered nonfatal, highly curable with rare metastases, and low recurrence with limited impact on mortality and morbidity $[27,28]$.

Recent studies of the effectiveness of MMS observed 5-year local control rates of $99 \%$ and 92-99\% for primary BCC and SCC, respectively; the corresponding rates for recurrent $\mathrm{BCC}$ and SCC treated with MMS are $90-93 \%$ and $90 \%$, respectively [3]. Comparison of the results of this study with those achievable using surgical therapies, such as MMS, may be limited. MMS is indicated for higher-risk NMSC lesions, and situations based on expert opinion, smaller lesions and/or lower risk lesions with cosmetic or functional significance as well [2, 29].
In this study, IGSRT was administered to a range of tumor types and sizes, not only highrisk lesions. It is notable, however, that the median tumor diameter in this study was $1.0 \mathrm{~cm}$, with an upper diameter of $5.5 \mathrm{~cm}$. This indicates that most of the NMSC lesions treated in this study were clinically significant tumors that would have required substantial excisions to achieve surgical eradication-not minute, easily curable lesions. While the results of this retrospective review of IGSRT patients demonstrate a consistent local tumor control rate in a limited follow-up period, retrospective comparisons of matched cohorts or prospective trials with IGSRT given in this manner with further follow-up for additional years would be helpful to evaluate any differences observed in IGSRT and MMS outcomes in the treatment of NMSC. A prospective randomized trial comparing IGSRT vs MMS would provide an ideal comparison; however, ethically and practically, it may prove difficult to conduct a study randomizing patients to a surgical vs non-surgical modality. In this study cohort, patients were given the option of surgical versus non-surgical IGSRT options and they chose the non-surgical method for various reasons that were not documented or extracted. Presumably, many patients may have opted for IGSRT to avoid the pain, scarring, and risk of infection or bleeding that may be associated with surgery.

Absolute and relative contraindications of IGSRT include invasion of underlying bone or muscle, thickness (greater than $6 \mathrm{~mm}$ ) that cannot be debulked, previous irradiation of the same site, ataxia telangiectasia, active connective tissue disease, active or uncontrolled lupus or rheumatologic conditions, concomitant administration of radiation sensitizing chemotherapy (i.e., doxorubicin), T4 or node positive status, amongst others.

The high rate of local tumor control suggests that the use of daily pre-treatment lesion ultrasound imaging and daily evaluation of tumor depth can improve delivery of the optimal radiation dose and energy over a fractionated treatment scheme. In this study, adjustments to the energy were typically performed after the 5 th, 10 th, or 15 th treatments on the basis of review of the ultrasound depth by the 
practitioner. In certain circumstances, a significant change in tumor depth can be detected from one fraction to another, depending on tumor progression or external factors (i.e., bleeding, injury, excoriation, scab formation, etc.) which can prompt the radiation therapist to alert the provider to change the energy in order to cover the entirety of the new tumor configuration accordingly. Thus, pre-treatment ultrasound is reasonable and recommended for this purpose, in addition to daily verification/ localization of the tumor. Indeed, $29 \%$ of the lesions treated utilized multiple energies, potentially due in part to the change in imaging characteristics (depth) encountered. Limitations of the ultrasound include that it cannot detect tumor deeper than $6 \mathrm{~mm}$ (though tumors invading beyond this depth are contraindicated for treatment with IGSRT). Additionally, ultrasound imaging is difficult to attain if the surface is irregular or actively bleeding, which does not allow adequate contact of the probe with the skin. Further study is warranted to evaluate the impact of pre-treatment ultrasound imaging on SRT efficacy, but an improvement in SRT efficacy with image guidance would be consistent with general principles and published results in radiation treatments at other sites of cancer that utilize image guidance and planning [30-32].

\section{Safety}

IGSRT was generally well tolerated and safe. Typical side effects of radiation to the skin, such as erythema, hyperpigmentation, dryness, edema, etc., consistent with historical SRT treatments were seen. RTOG toxicity grades were recorded for 2177 (74.6\%) of lesions treated. ${ }^{3}$ Among 2177 lesions, grade 3 and grade 4 toxicities were documented in only $16(0.5 \%)$ and $4(0.1 \%)$ lesions, respectively. This overall low level of toxicity is in accordance with prior literature indicating that radiation therapy for NMSC is generally well tolerated, with acute

\footnotetext{
3 Certain study locations did not maintain a procedure to record RTOG toxicity grades for IGSRT patients in the initial study year(s), accounting for the missing values on this measure of safety.
}

reactions being site-specific and self-limited [33].

\section{CONCLUSIONS}

IGSRT treatment for NMSC in a total of 1632 patients (total of 2917 NMSC lesions) in this study is well tolerated, achieves excellent local tumor control, and should continue to be recommended in the management of this disease. Among these patients, 55\% were followed for a minimum of 12 months. Use of pre-treatment ultrasound and ongoing imaging to adjust radiation energy and dose, combined with a fractionation treatment schedule of 20 or more treatment fractions, has the potential to be associated with improved local tumor control in NMSC lesions compared to SRT without ultrasound imaging.

IGSRT is a safe and highly effective modality for treating NMSC. It should be considered as a first-line option for stage 0-II NMSC lesions (including for tumors less than $4 \mathrm{~cm}$ in diameter and/or SCCIS with substantial, full-thickness atypia of the epidermis or with strong clinical suspicion of invasion), especially in those patients who are not candidates for surgery or who decline surgery.

\section{ACKNOWLEDGEMENTS}

The authors would like to acknowledge the following people who were instrumental in collecting and extracting data from various different locations, without whom this study would not be possible: Amanda Boatner, Charles Bingner, Jacqueline Contino, Olivia Desalvo, Lisa Gonzalez, Peter Kaczmarksi, Jolanta Kacznarski, Michael Kacznarski, Erica Kovaluskie, Rachel Lindley, Carolyn Mahoot, Kim McLaugherty, Mairead Moloney, Sarah Moss, Nick Natalizio, Krupal Patel, Samantha Sheehan, Kimberly Wilke-Zuma, Paula Wright, and Soham More and Prithvi Narasimhan from Sympto Health, Inc. We extend special thanks to Mark Jaros, PhD and Michelle Secic of Summit Analytical for invaluable assistance in 
statistical direction and analyses. We also thank the participants of this study

Funding. This study was sponsored by SkinCure Oncology, including the journal's Rapid Service Fee.

Authorship. All named authors meet the International Committee of Medical Journal Editors (ICMJE) criteria for authorship for this article, take responsibility for the integrity of the work as a whole, and have given their approval for this version to be published.

Authorship Contributions. All authors contributed in the development of this article. Data collection and analysis were performed primarily by Dr. Yu, with assistance from Dr. Oh. The first draft of the manuscript was shared equally among the authors, and all authors commented on previous versions of the manuscript. All authors read and approved the final manuscript.

Disclosures. Dr. Yu serves as the National Radiation Oncologist for SkinCure Oncology and has received research, speaking, and/or consulting support from SkinCure Oncology. Dr. Oh has received research support from SkinCure Oncology. Dr. Shea has received research, speaking, and/or consulting support from a variety of companies including Myriad Genetics, Novartis, Orlucent, and SkinCure Oncology.

Compliance with Ethics Guidelines. The authors adhered to the principles established in the Federal Policy for the Protection of Human Subjects, referred to as the "Common Rule," as well as the pertinent sections of the Helsinki Declaration and its amendments. The data have been de-identified for use in this study. All patients gave informed consent before treatment.

Data Availability. Data are available on request from the authors.

Open Access. This article is licensed under a Creative Commons Attribution-
NonCommercial 4.0 International License, which permits any non-commercial use, sharing, adaptation, distribution and reproduction in any medium or format, as long as you give appropriate credit to the original author(s) and the source, provide a link to the Creative Commons licence, and indicate if changes were made. The images or other third party material in this article are included in the article's Creative Commons licence, unless indicated otherwise in a credit line to the material. If material is not included in the article's Creative Commons licence and your intended use is not permitted by statutory regulation or exceeds the permitted use, you will need to obtain permission directly from the copyright holder. To view a copy of this licence, visit http:// creativecommons.org/licenses/by-nc/4.0/.

\section{REFERENCES}

1. Rogers HW, Weinstock MA, Feldman SR, Coldiron $\mathrm{BM}$. Incidence estimate of nonmelanoma skin cancer (keratinocyte carcinomas) in the U.S. population, 2012. JAMA Dermatol. 2015;151(10): 1081-6. https://doi.org/10.1001/jamadermatol. 2015.1187.

2. Ad Hoc Task Force, Connolly SM, Baker DR, et al. AAD/ACMS/ASDSA/ASMS 2012 appropriate use criteria for Mohs micrographic surgery: a report of the American Academy of Dermatology, American College of Mohs Surgery, American Society for Dermatologic Surgery Association, and the American Society for Mohs Surgery [published correction appears in J Am Acad Dermatol. 2015 72(4):748]. J Am Acad Dermatol. 2012;67(4):531-550. https:// doi.org/10.1016/j.jaad.2012.06.009.

3. Tolkachjov SN, Brodland DG, Coldiron BM, et al. Understanding Mohs micrographic surgery: a review and practical guide for the nondermatologist. Mayo Clin Proc. 2017;92(8):1261-71. https:// doi.org/10.1016/j.mayocp.2017.04.009.

4. Rowe DE, Carroll RJ, Day CL Jr. Mohs surgery is the treatment of choice for recurrent (previously treated) basal cell carcinoma. J Dermatol Surg Oncol. 1989;15(4):424-31. https://doi.org/10.1111/j.15244725.1989.tb03249.x.

5. Mori WS, Demer AM, Mattox AR, Maher IA. Mohs micrographic surgery at challenging anatomical 
sites. Dermatol Surg. 2019;2(45 Suppl):142-54. https://doi.org/10.1097/DSS.0000000000002255.

6. Bowen GM, White GL Jr, Gerwels JW. Mohs micrographic surgery. Am Fam Physician. 2005;72(5):845-8.

7. Newlands C, Currie R, Memon A, Whitaker S, Woolford T. Non-melanoma skin cancer: United Kingdom National Multidisciplinary Guidelines. J Laryngol Otol. 2016;130(S2):S125-32. https://doi. org/10.1017/S0022215116000554.

8. Lang PG Jr. The role of Mohs' micrographic surgery in the management of skin cancer and a perspective on the management of the surgical defect. Clin Plast Surg. 2004;31(1):5-31. https://doi.org/10. 1016/s0094-1298(03)00096-8.

9. Patel R, Strimling R, Doggett S, et al. Comparison of electronic brachytherapy and Mohs micrographic surgery for the treatment of early-stage non-melanoma skin cancer: a matched pair cohort study. J Contemp Brachytherapy. 2017;9(4):338-44. https://doi.org/10.5114/jcb.2017.68480.

10. Silva JJ, Tsang RW, Panzarella T, Levin W, Wells W. Results of radiotherapy for epithelial skin cancer of the pinna: the Princess Margaret Hospital experience, 1982-1993. Int J Radiat Oncol Biol Phys. 2000;47(2):451-9. https://doi.org/10.1016/s03603016(00)00410-7.

11. Work Group; Invited Reviewers, Kim JYS, Kozlow $\mathrm{JH}$, et al. Guidelines of care for the management of basal cell carcinoma. J Am Acad Dermatol. 2018;78(3):540-59. https://doi.org/10.1016/j.jaad. 2017.10.006.

12. Hernández-Machin B, Borrego L, Gil-García M, Hernández $\mathrm{BH}$. Office-based radiation therapy for cutaneous carcinoma: evaluation of 710 treatments. Int J Dermatol. 2007;46(5):453-9. https:// doi.org/10.1111/j.1365-4632.2006.03108.x.

13. Cognetta AB, Howard BM, Heaton HP, Stoddard ER, Hong HG, Green WH. Superficial x-ray in the treatment of basal and squamous cell carcinomas: a viable option in select patients. J Am Acad Dermatol. 2012;67(6):1235-41. https://doi.org/10.1016/j. jaad.2012.06.001.

14. National Comprehensive Cancer Network. Basal Cell Skin Cancer (Version 1.2020). https://www. nccn.org/professionals/physician_gls/pdf/nmsc. pdf. Accessed Aug 28, 2020.

15. National Comprehensive Cancer Network. Squamous Cell Skin Cancer (Version 2.2020). https:// www.nccn.org/professionals/physician_gls/pdf/ squamous.pdf. Accessed Aug 28, 2020.
16. Kasper M, Chaudhary A. Novel treatment options for nonmelanoma skin cancer: focus on electronic brachytherapy. Med Devices (Auckl). 2015;8: 493-502. https://doi.org/10.2147/MDER.S61585.

17. Thomas KS, Batchelor JM, Bath-Hextall F, et al. A programme of research to set priorities and reduce uncertainties for the prevention and treatment of skin disease. Programme Grants Appl Res. 2016;4: 196-7.

18. Melton LJ. Selection bias in the referral of patients and the natural history of surgical conditions. Mayo Clin Proceed. 1985;60:880-5. https://doi.org/10. 1016/S0025-6196(12)64794-6.

19. U.S. Food and Drug Administration. SRT-100 Vision 510(k) Clearance Letter (K150037). https://www. accessdata.fda.gov/scripts/cdrh/cfdocs/cfpmn/ pmn.cfm. Accessed Aug 31, 2020.

20. Yu L, Ladd D. 555 Practice changing landmark study- multi-institutional analysis of image guided superficial radiotherapy (IGSRT) for the treatment of non-melanoma skin cancer (NMSC). J Investig Dermatol. 2020;140(7):S75. https://doi.org/10. 1016/j.jid.2020.03.564.

21. Edge SB, Edge SB, Committee AJ, on Cancer. AJCC cancer staging manual. 8th ed. New York: Springer; 2017.

22. Mendenhall WM, Dagan R, Bryant CM, Amdur RJ. Definitive radiotherapy for skin and adenoid cystic carcinoma with perineural invasion. J Neurol Surg B Skull Base. 2016;77(2):169-72. https://doi.org/10. 1055/s-0036-1579545.

23. Chan S, Dhadda AS, Swindell R. Single fraction radiotheray for small superficial carcinoma of the skin. Clin Oncol (R C Radiol). 2007;19:256-9. https://doi.org/10.1016/j.clon.2007.02.004.

24. Likhacheva A, Awan M, Barker CA, et al. Definitive and postoperative radiation therapy for basal and squamous cell cancers of the skin: executive summary of an American Society for Radiation Oncology clinical practice guideline. Practl Rad Oncol. 2020;10(1):8-20. https://doi.org/10.1016/j.prro. 2019.10.014.

25. Cox JD, Stetz J, Pajak TF. Toxicity criteria of the Radiation Therapy Oncology Group (RTOG) and the European Organization for Research and Treatment of Cancer (EORTC). Int J Radiat Oncol Biol Phys. 1995;31(5):1341-6. https://doi.org/10.1016/ 0360-3016(95)00060-C.

26. Xu JQ, Murphy SL, Kochanek KD, Arias E. Mortality in the United States, 2018. NCHS Data Brief, no. 355. Hyattsville, MD: National Center for Health Statistics. 2020. 
27. Linos E, Parvataneni R, Stuart SE, Boscardin WJ, Landefeld CS, Chren MM. Treatment of nonfatal conditions at the end of life: nonmelanoma skin cancer. JAMA Intem Med. 2013;173(11):1006-12. https://doi.org/10.1001/jamainternmed.2013.639.

28. Zagrodnik B, Kempf W, Seifert B, et al. Superficial radiotherapy for patient with basal cell carcinoma: recurrence rates, histologic subtypes, and expression of p53 and Bcl-2. Cancer. 2003;98(12): 2708-14. https://doi.org/10.1002/cncr.11798.

29. Murray C, Sivajohanathan D, Hanna TP, et al. Patient indications for Mohs micrographic surgery: a systematic review. J Cutan Med Surg. 2019;23(1): 75-90. https://doi.org/10.1177/ 1203475418786208.

30. Bohrer M, Schröder P, Welzel G, et al. Reduced rectal toxicity with ultrasound-based image guided radiotherapy using BAT (B-mode acquisition and targeting system) for prostate cancer. Strahlenther Onkol. 2008;184(12):674-8. https://doi.org/10. 1007/s00066-008-1837-z.

31. Sayan M, Vergalasova I, Hard D, et al. Daily localization of partial breast irradiation patients with three-dimensional ultrasound imaging. Rad Oncol J. 2019;37(4):259-64. https://doi.org/10.3857/roj. 2019.00052 .

32. Western C, Hristov D, Schlosser J. Ultrasound imaging in radiation therapy: from interfractional to intrafractional guidance. Cureus. 2015;7(6):e280. https://doi.org/10.7759/cureus.280.

33. Veness MJ, Delishaj D, Barnes EA, Bezugly A, Rembielak A. Current role of radiotherapy in non-melanoma skin cancer. Clin Oncol (R Coll Radiol). 2019;31(11):749-58. https://doi.org/10.1016/j. clon.2019.08.004. 